\title{
NGHIÊN CÚU THƯỚC ĐO GIÁ TRI KINH TẾ GIA TĂNG (ECONOMIC VALUE ADDED- EVA) VẦ CÁC VẤN ĐỀ LIÊN QUAN
}

\author{
NGUYỄN NGỌC KHÁNH DUNG \\ Trường Đại học Công nghiệp Thành phố Hồ Chí Minh \\ khanhdung1608@yahoo.com.vn
}

Tóm tắt. Giá trị kinh tế gia tăng (EVA) được sử dụng để đánh giá thành quả hoạt động của nhà quản lý. Kể từ khi công cụ này được công bố tới nay, có nhiều doanh nghiệp tại các quốc gia phát triển đã triển khai ứng dụng và đạt được những thành tựu nhất định. Lần đầu tiên (theo quan điểm của một số nhà nghiên cứu), thước đo này đã trở thành "thủ lĩnh tiên phong" trên "mặt trận" đo lường hiệu quả tài chính của doanh nghiệp nhưng một số nhà nghiên cứu khác thì không đồng thuận như vậy. Bài viết nhằm mục đích khái quát hóa các quan điểm lý luận cũng như kinh nghiệm thực tiễn ứng dụng thước đo EVA trên thế giới, qua đó giúp định hình xu thế và khả năng vận dụng trong thực tiễn hoạt động quản trị doanh nghiệp cũng như nghiên cứu ở Việt Nam. Phương pháp tổng quan nghiên cứu được thực hiện thông qua lược đồ phân loại các dòng nghiên cứu theo thời gian, các vấn đề nghiên cứu đã được giải quyết, các vấn đề còn gây tranh cãi để gợi ý hướng nghiên cứu tiếp theo. Nghiên cứu này kế thừa nghiên cứu của Anil K. Sharma và Satish Kumar (2008) đến 2008 và tổng kết bổ sung các nghiên cứu về EVA đã thực hiện từ năm 2009 đến 2017. Tù khóa. Giá trị kinh tế gia tăng, giá trị thị trường gia tăng, thước đo truyền thống, Thu nhập sau thuế và lãi vay, Thu nhập trên vốn chủ sở hữu.

\section{ECONOMIC VALUE ADDED (EVA) - LITERATURE REVIEW AND RELEVANT ISSUES}

\begin{abstract}
Economic Value Added is recognized as an important tool of performance measurement and management. This tool is introduced by Stern Stewart \& Co trademarks in 1990s and subsequently adopted by several major corporations. EVA is a better performance measures than other performance measures from the point of view of many literatures. However, some studies reject the superiority of EVA partially or fully. So, present study has been conducted to find out various issues underlying EVA. This study aims to summarize theoretical perspectives as well as practical experience applying the EVA in the world, thereby helping to shape the trend and ability to apply in practice corporate governance as well as study in Viet Nam. This study provides a classification scheme, identifies the gaps in existing literature and suggests the direction for future research. The research method used in this study is almost identical to that used in Anil K. Sharma \& Satish Kumar (2008) which review of 112 papers published on the EVA from 1994 to 2008 and additional review of EVA studies conducted from 2009 to 2017.
\end{abstract}

Keywords. EVA, Market Value Added, NOPAT, Traditional measures, ROE.

\section{GIỚI THIỆ}

Giá trị kinh tế gia tăng (EVA) được nhìn nhận là một trong những thước đo thành quả tài chính hữu ích nhất (Brewer, Chandra và Hock, 1999; Fatemi, Desai, và Katz, 2003; Fiordelisi, Stefano và Monferrà, 2009; Fiordelisi và Molyneux, 2010; Kryzanowski và Mohsni, 2010; Lee và Kim, 2009; Nam và cộng sự, 2009; Stern, Stewart và Chew, 1991; Yao và cộng sự, 2009; Young và O'Byrne, 2000; Young, 2010). Ngoài chức năng đánh giá hiệu quả hoạt động như thước đo truyền thống, EVA còn giúp xác định lợi ích tăng thêm mà cổ đông được hưởng. Madhavi và Prasad (2015: 19) nhấn mạnh "EVA chỉ ra lợi nhuận thực sự của công ty", điều mà các cồ đông luôn muốn biết. Điểm mấu chốt khiến EVA nhận được hiệu ứng tích cực hơn các thước đo truyền thống khác như NOPAT, EPS, ROI, ROE là do EVA sử dụng các thông số kinh tế để điều chỉnh các số liệu kế toán và có tính đến chi phí sử dụng vốn (Johnson và Kaplan, 1987; Kaplan và Norton, 1996; Olve, Roy và Wetter, 1999).

Trường phái ủng hộ việc ứng dụng EVA thay thế các thước đo truyền thống khác trong đánh giá thành quả hoạt động của doanh nghiệp dựa trên bốn lập luận căn bản như (i) EVA giúp giảm xung đột lợi ích giữa 
các bên, đồng thời giúp cải thiện hiệu quả ra quyết định (Costigan và Lovata, 2002; Biddle và cộng sự, 1999); (ii) EVA có tương quan chặt chẽ hơn với lợi tức cổ phiếu so với các thước đo truyền thống (Maditinos và cộng sự 2006; Lehen và Makhija, 1997); (iii) EVA có tác động tích cực đến lợi tức cổ phiếu (Ferguson và cộng sự, 2005); (iv) cung cấp thêm thông tin về giá trị kinh tế tăng thêm cho cổ đông cũng như giải thích rõ ràng hơn về cố tức và giá trị cổ phiếu (Erasmus, 2008; Chen và Dodd, 1997; Kim, 2006; Palliam, 2006); (v) EVA và giá trị thị trường có mối tương quan (Lefkowitz, 1999; O'Byrne, 1996; Uyemura, 1996; Peterson và Peterson, 1996).

Trường phái phản bác vai trò và tầm quan trọng của EVA cho rằng (i) EVA không có tương quan với lợi tức cổ phiếu so với các thước đo truyền thống (Biddle và cộng sự, 1997, 1999); (ii) lợi nhuận thuần sau thuế và thu nhập hoạt động thuần là hai chỉ số đo lường hữu ích hơn EVA trong việc giải thích lợi tức cổ phiếu (Nicolás Cachanosky và Peter Lewin, 2016; Ismail, 2006); (iii) chỉ số thu nhập hoạt động thuần hữu ích hơn EVA trong việc đánh giá thành quả hoạt động (Kyriazis và Anastassis, 2007).

\section{THƯỚC ĐO GIÁ TRI KINH TẾ GIA TĂNG (EVA)}

\section{Khái niệm}

EVA xuất hiện từ đầu thập niên 1990 (Bromwich và Walker, 1998, p. 392), nhưng được đặt tên, đăng ký nhãn hiệu thương mại bởi Stern Stewart \& Co. Sau khi giới thiệu, quảng bá thước đo này, đã có nhiều tập đoàn, công ty lớn áp dụng để đánh giá thành quả của doanh nghiệp cũng như kết quả công việc của nhà quản lý. Điểm khác biệt lớn nhất giữa EVA và các thước đo truyền thống là cách xác định và tính toán lợi nhuận. Các thước đo truyền thống xác định lợi nhuận dựa trên thông số tài chính, trong khi lợi nhuận sử dụng để tính EVA là lợi nhuận kinh tế (có tính đến chi phí vốn và các tỷ số chiết khấu theo thị trường).

\section{Tính toán EVA}

EVA là phần còn lại khi lấy lợi nhuận hoạt động thuần sau thuế trừ (-) chi phí sử dụng vốn, công thức:

EVA = NOPAT - (TCE $x$ WACC) (1)

Trong đó:

NOPAT = Lợi nhuận hoạt động thuần sau thuế

TCE $=$ Tổng vốn được sử dụng

WACC = Chi phí vốn bình quân gia quyền

Khi tính toán NOPAT, các hoạt động không liên quan đến hoạt động sản xuất kinh doanh như hoạt động đầu tư, góp vốn, tài chính hoặc các khoản chi phí phát sinh không liên quan đến hoạt động sản xuất kinh doanh sẽ được loại trừ. Tổng vốn được sử dụng bao gồm số vốn góp của cổ đông, và các khoản vay nhưng không gồm các khoản đầu tư ra bên ngoài doanh nghiệp. Khi xác định WACC, chi phí nợ được tính sau thuế và chi phí vốn chủ sở hữu được xác định bằng phương pháp định giá tài sản vốn. Theo mồ hình định giá tài sản vốn, chi phí vốn chủ sở hữu $(\mathrm{Ke})$ được đưa ra như sau:

\section{$\mathrm{Ke}=\mathbf{R f}+\mathbf{b i}(\mathbf{R m}-\mathbf{R f})(2)$}

Trong đó:

$$
\begin{aligned}
& \mathrm{Rf}=\text { Lãi suất phi rủi ro } \\
& \mathrm{Rm}=\text { Lãi suất thị trường mong muốn } \\
& \text { bi = Hệ số rủi ro của đầu tư }
\end{aligned}
$$

Chi phí sử dụng vốn là yếu tố quan trọng nhất trong tính toán EVA, bởi EVA có tính tới chi phí sử dụng vốn khi đánh giá thành quả hoạt động của doanh nghiệp hay còn được gọi chi phí cơ hội của vốn đầu tư. Khi đánh giá thành quả hoạt động theo phương pháp truyền thống, hầu hết các công ty đều có lợi nhuận, nhưng mức lợi nhuận có thể thấp hơn việc đầu tư vào các doanh nghiệp tương tự hoặc lĩnh vực khác. Peter Drucker (1995, p.59) khẳng định rằng "Khi nào lợi nhuận của doanh nghiệp lớn hơn chi phí của việc sử dụng vốn thì doanh nghiệp mới thực sự có lời. Ngược lại, nó không tạo ra sự giàu có mà còn làm xói mòn chúng" 


\section{CÁC DÒNG NGHIÊN CÚU VỀ EVA ĐƯợC THỰC HIỆN TỪ 1994 ĐẾN NAY}

\subsection{Các dòng nghiên cứu chính}

Tổng kết từ nghiên cứu của Anil K. Sharma và Satish Kumar (2008) và các nghiên cứu từ 1994 đến nay (Bảng 1) có thể phân loại nghiên cứu về EVA thành 7 dòng nghiên cứu dựa trên 7 nội dung trọng tâm cần phải khám phá, giải thích. Từ năm 2009 tới nay, có thêm một dòng nghiên cứu mới là các ứng dụng phát triển của EVA trong thực tiễn. Các dòng nghiên cứu chính được phân loại như Hình 1.

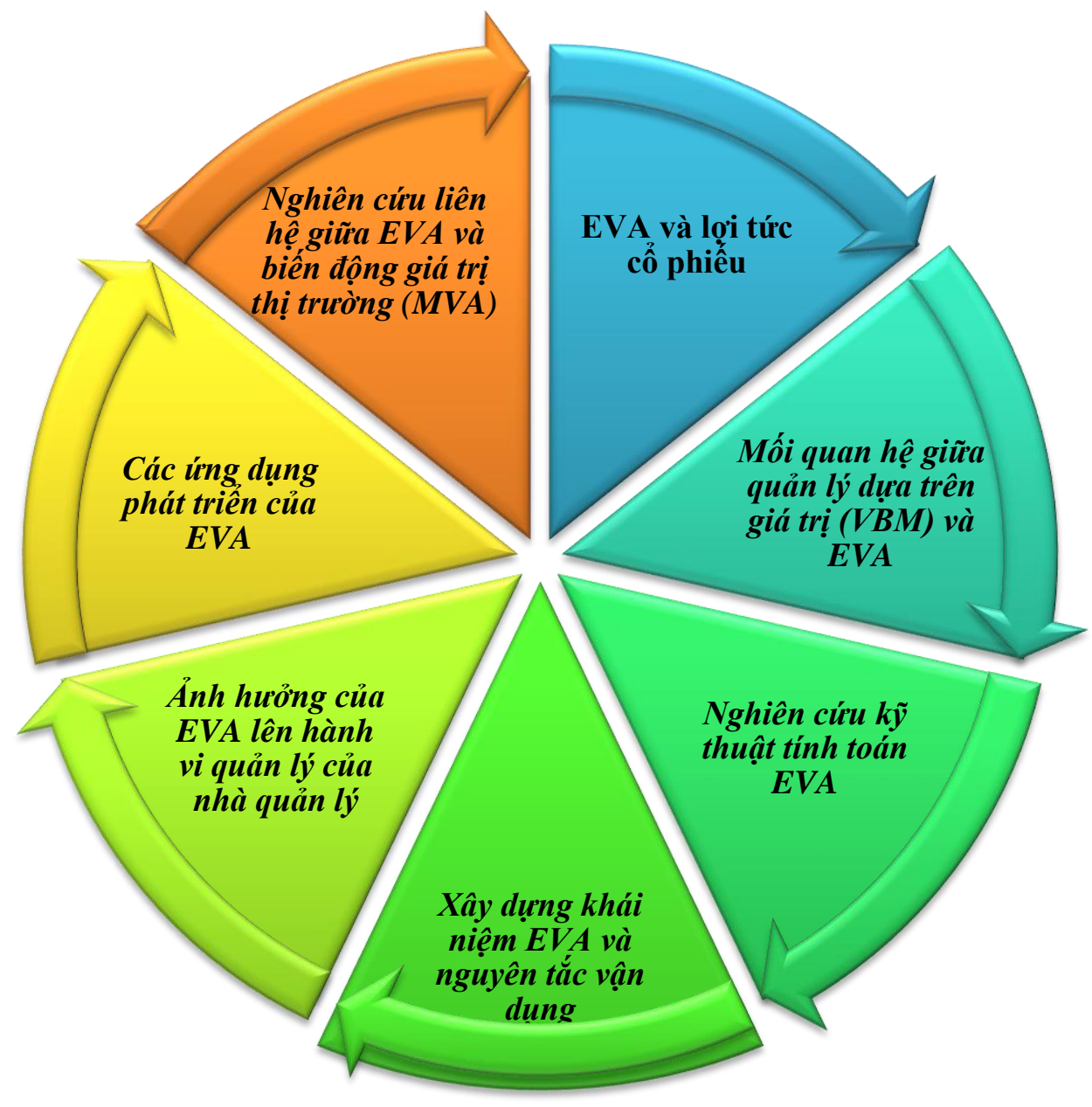

Hình 1: Các dòng nghiên cứu về EVA (Tác giả tự tổng kết)

(1) Nghiên cúu liên hệ giữa EVA và biến động giá trị thị trường (MVA): Các nghiên cứu này thực hiện điều tra, khảo sát nhằm chứng tỏ liệu thông điệp EVA cung cấp có tác động đến giá trị thị trường và mức độ tác động. Điển hình là các nghiên cứu của Fiordelisi và Molyneux (2010), Lee và Kim (2009), Man và Vasile (2009) và Stewart (2014).

(2) Nghiên cúu tương quan giữa EVA và lợi tức cổ phiếu: Chủ đề này nhận được sự quan tâm, chú ý nhiều nhất trong giới nghiên cứu. Các nghiên cứu này tập trung khảo sát nhằm so sánh EVA với các thước đo truyền thống khác, cung cấp bằng chứng thực nghiệm chứng tỏ mối tương quan giữa EVA với lợi tức cổ phiếu, qua đó giải thích tác động cũng như tầm quan trọng của thước đo này (Saji, 2014; Cendrowski, 2013; Kryzanowski và Mohsni, 2010; Yao, Sutton và Chan, 2009; Fiordelisi, Stefano và Monferrà, 2009; Tortella và Brusco, 2003). Bên cạnh đó, các nghiên cứu thực hiện kiểm chứng tính hữu ích của thông tin, khả năng dự báo thu nhập của EVA so với thước đo thu nhập tăng thêm (RI). 
(3) Nghiên cúu mối quan hệ giữa quản lý dụa trên giá trị (VBM) và EVA: Nội dung các nghiên cứu này tập trung vận dụng EVA trong các tình huống khác nhau, chẳng hạn, EVA sử dụng để định giá chi phí hoạt động hoặc cung cấp thông tin hữu ích cho việc ra quyết định hoặc sử dụng để xây dựng, ban hành, và điều chỉnh các chính sách cụ thể nhằm cải thiện kết quả kinh doanh (Austin, 2005; Young và O'Byrne, 2000; Nam và cộng sự, 2009; Altendorfer và Jodlbauer, 2011). Tương tự, Foshee (2000) khẳng định EVA định hướng việc ra quyết định quản lý, quyết định tài chính của công ty nhằm gia tăng giá trị cho các tổ chức.

(4) Nghiên cứu $k \tilde{y}$ thuật tính toán EVA: Việc xác định tỷ suất chiết khấu nhằm mục đích đưa dòng tiền tương lai ở các năm khác nhau về thời điểm hiện tại để loại bỏ ảnh hưởng của yếu tố thời gian. Nếu dự án đầu tư chỉ sử dụng vốn tự có, tỷ suất chiết khấu này được xác định bằng tỷ suất sinh lợi của cơ hội đầu tư ít rủi ro nhất, thường là lãi suất gửi ngân hàng hoặc lãi suất trái phiếu Chính phủ. Nếu dự án đầu tư chỉ sử dụng vốn vay, tỷ suất chiết khấu được xác định bằng lãi suất tiền vay. Thực tế thì các dự án thường sử dụng cả hai nguồn vốn này, lúc này, tỷ suất chiết khấu thường được xác định bằng phương pháp bình quân gia quyền dựa trên tỷ trọng cơ cấu vốn. Do đó, các nghiên cứu này tập trung tìm hiểu cách thức xác định EVA trong các tình huống áp dụng tỷ suất chiết khấu khác nhau (Shrieves và Wahowicz, 2001), chẳng hạn, trong việc xác định giá trị hiện tại ròng (NPV) hoặc tính EVA có điều chỉnh lạm phát và thu nhập còn lại (Burksaitiene, 2009; Saeedi và Akbari, 2010; De Villiers, 1997).

(5) Nghiên cúu xây dụng khái niệm EVA và nguyên tắc vận dụng: Nội dung của các nghiên cứu này gồm: xem xét EVA như hệ thống quản lý tài chính, xây dựng chiến lược, kỹ thuật tính toán EVA, những ưu điểm và hạn chế khi vận dụng EVA như nghiên cứu của Yao, Sutton và Chan (2009); Satish và Rau (2009); Saeedi và Akbari (2010) và De Villiers (1997). Ngoài ra, Ehrbar (1999) và Rompho (2009) cho rằng EVA là một công cụ lý tưởng mà các công ty có thể sử dụng để tối ưu ngân sách và lập kế hoạch chiến lược.

(6) Nghiên cúu thục nghiệm ảnh hưởng của EVA lên hành vi quản lý của nhà quản lý: Nội dung các nghiên cứu này tập trung xem xét tác động EVA lên hành vi nhà quản lý cũng như việc thiết kế, xây dựng chính sách khen thưởng của công ty. Giả thuyết trong các nghiên cứu này cho rằng, khi sử dụng $\mathrm{EVA}$ là công cụ đánh giá thành quả hoạt động, các nhà quản lý sẽ có động lực cao hơn trong việc nâng cao năng suất hoạt động và hiệu quả sử dụng vốn (Desai và Ferri, 2006; Young, 2010; Mittal, Sinha và Singh, 2008; Ghani, Tezel, Ragan và Stagliano, 2005; Rodgers, 2007; Bostan và cộng sự, 2010; Young, 1997; Murphy, 2007). Poornima, Narayan và Reddy (2015) cho rằng, EVA ngày càng được sử dụng phổ biến trong việc đánh giá thành quả quản lý. Tương tự, Bahri, St-Pierre và Sakka (2011: 603) khẳng định rằng "EVA có thể là một công cụ hữu ích để quản lý hiệu quả hoạt động của doanh nghiệp vừa và nhỏ khi được sử dụng kết hợp với các phương thức quản lý kinh doanh khác". Các phương thức này gồm quản lý bán hàng, quản lý sản xuất, quản lý tài chính, quản lý thiết bị sản xuất và quản lý vốn lưu động. Trong khi đó, Hamilton và cộng sự (2009) nhấn mạnh rằng EVA được sử dụng cho cả lĩnh vực tổ chức nhân sự và cải thiện hiệu suất của tổ chức.

(7) Nghiên cứu các úng dụng phát triển của EVA: gồm các nghiên cứu về việc ứng dụng EVA cho những mục đích quản trị khác trong doanh nghiệp, chẳng hạn nghiên cứu của Shad và Lai (2015) đưa ra khung khái niệm để phát triển các biện pháp quản lý rủi ro doanh nghiệp thông qua thước đo EVA; nghiên cứu của Qin Chen và XiaoMei Zhang (2011) đề xuất Thẻ cân bằng điểm (BSC) được đánh giá dựa trên EVA.

\subsection{Các phương pháp nghiên cứu}

Tổng kết từ nghiên cứu của Sharma và Kumar (2008) và 16 nghiên cứu từ 2009 đến nay cho thấy có bốn phương pháp luận nghiên cứu chính gồm (i) nghiên cứu lý thuyết (Conceptual), (ii) nghiên cứu mô tả (Descriptive), (iii) nghiên cứu thực nghiệm (Empirical), (iv) nghiên cứu khám phá (Exploratory crosssectional). Các nghiên cứu lý thuyết nhằm xây dựng khái niệm EVA, điển hình nghiên cứu của Martin và cộng sự (2003), Mohanty (2006), Phillips (2007). Nghiên cứu mô tả nhằm giải thích, mô tả EVA và cách thức ứng dụng EVA trong thực tiễn, ví dụ nghiên cứu của Keef và Roush (2003), Dodd và Chen (1996), Paulo (2002),...v.v. Phương pháp nghiên cứu được thực hiện qua điều tra, khảo sát các doanh nghiệp, kết hợp việc phỏng vấn các nhà quản lý, người sở hữu vốn, nổi bật là nghiên cứu của Athanassakos (2007), Nappi-Choulet và cộng sự (2007), Taufik và cộng sự (2008). Nghiên cứu khám phá nhằm phát hiện, tìm hiểu, giải thích và đo lường các yếu tố tác động đển việc vận dụng EVA và ảnh hưởng của EVA đến hành 
vi của nhà quản lý, các cổ đông như nghiên cứu của Lefkowitz (1999), Weaver (2001), Kumar và Pal (2008).

So với tổng kết nghiên cứu của Anil K. Sharma và Satish Kumar (2008), các nghiên cứu được công bố sau này không chỉ tập trung khảo sát nhằm so sánh EVA với các thước đo truyền thống khác và cung cấp bằng chứng thực nghiệm chứng tỏ mối tương quan giữa EVA với lợi tức cổ phiếu, v.v, mà còn tập trung nghiên cứu các ứng dụng phát triển của EVA cũng như việc kết hợp EVA và các thước đo khác là một cách tiếp cận chung nhất giúp nhà quản lý phát hiện những vấn đề phát sinh và xác định nguồn lực cho sự phát triển của doanh nghiệp.

\subsection{Các phát hiện chính trong các nghiên cứu EVA.}

EVA là chủ đề quan tâm của các nhà nghiên cứu đặc biệt ở Mỹ, Nam Phi, Anh và một số quốc gia phát triển khác từ năm 1994 đến nay. Phần lớn các nghiên cứu liên quan đến EVA và sự thay đổi giá của cổ phiếu hoặc so sánh EVA với các thước đo truyền thống nhằm đánh giá thành quả hoạt động của doanh nghiệp. Hầu hết các nhà nghiên cứu, giới nghề nghiệp đều thừa nhận rằng, EVA là một thước đo hữu hiệu trong đánh giá thành quả hoạt động của doanh nghiệp cũng như trách nhiệm nhà quản lý và nếu hoàn thiện chỉ tiêu này có thể đại diện cho hầu hết các chỉ số tài chính truyền thống trước đây, cung cấp thông tin về lợi ích kinh tế xác thực hơn cho các cổ đông, nhà đầu tư. Nếu được phổ biến rộng rãi, thước đo này không những được sử dụng trong việc thiết kế các chính sách lương, chính sách khen thưởng và có tác động nhất định đến các quyết định đầu tư, kinh doanh, quản lý và ảnh hưởng đến nhận thức và hành vi của nhà quản lý, nhà đầu tư...Tuy nhiên, vẫn tồn tại quan điểm khác biệt cả về lý luận lẫn thực tiễn khi vận dụng EVA liên quan đến các kỹ thuật tính toán xác định EVA và còn nhiều dư địa cho việc vận dụng EVA trong các lĩnh vực kinh tế, tài chính khác nhau, cho đến nay vẫn chưa được nghiên cứu đầy đủ.

\section{KHOẢNG TRỐNG TRONG NGHIÊN CÚU VỀ EVA}

\subsection{Kỹ thuật điều chỉnh số liệu kế toán để tính toán EVA}

Dữ liệu sử dụng cho việc tính toán EVA là số liệu kế toán thể hiện trên báo cáo tài chính, trong khi đó, EVA muốn hướng đến kết quả là dòng tiền thực, vì vậy, khi xác định lợi nhuận hay vốn đầu tư, cần phải thực hiện các điều chỉnh để loại bỏ những ghi chép đơn thuần về mặt kế toán. Để EVA phản ánh chính xác nhất thành quả, theo Stern- Stewart cần phải thực hiện 164 loại điều chỉnh trên báo cáo tài chính để xác định lợi nhuận ròng sau thuế (Stewart, 1991; 1994; 1999). Tuy nhiên, do tính chất phức tạ̣p trong cách thức điều chỉnh các khoản mục và mối tương quan với tính thích hợp của thông tin, nên vẫn còn nhiều quan điểm khác biệt về sự cần thiết, tính khoa học trong việc lựa chọn và cách thức điều chỉnh, chẳng hạn, việc loại bỏ một số khoản dự phòng để tính lợi nhuận ròng sau thuế một mặt làm cho EVA chính xác hơn nhưng một mặt làm giảm tính thích hợp của thổng tin. Đây chính là yếu tố cốt lõi quyết định sự hài hòa trong việc chọn lựa các kỹ thuật điều chỉnh (Worthington và West, 2001).

\subsection{Nghiên cứu và vận dụng EVA ở các nước đang phát triển}

Tổng kết các nghiên cứu vể EVA trên thế giới đã cho thấy các thông tin liên quan đến EVA đã được biết đến và được nghiên cứu ở các nền kinh tế tiên tiến như Mỹ, Úc, Brazil, Nam Phi trong khi đó các nền kinh tế đang phát triển có ít các nghiên cứu hơn về EVA được công bố. Đây có thể là một lĩnh vực nghiên cứu trong tương lai, đặc biệt là tại các nước đang phát triển nhằm cung cấp bằng chứng về ưu thế vượt trội của EVA so với các chỉ tiêu đánh giá thành quả hoạt động truyền thống.

\subsection{Nghiên cứu EVA theo từng nhóm ngành.}

Các doanh nghiệp được lựa chọn làm đối tượng khảo sát khi thực hiện nghiên cứu về EVA và các thước đo hoạt động truyền thống thường là các doanh nghiệp hoạt động sản xuất kinh doanh trong nhiều ngành nghề, lĩnh vực khác nhau. Do đó, những nghiên cứu này không cung cấp bằng chứng rõ ràng là thước đo nào hữu ích hơn trong việc giải thích lợi tức của cổ phiếu và có thể được sử dụng làm thước đo thành quả hoạt động. Sử dụng EVA và các thước đo truyền thống để phân tích và so sánh các kết quả các doanh nghiệp theo từng ngành có thể là một hướng mới trong nghiên cứu về EVA để cung cấp các bằng chứng về sự tồn tại và hữu hiệu của EVA trên phương diện thực tiễn. 


\subsection{Nghiên cứu việc ứng dụng EVA trong các ngành khác}

Từ tổng kết các phát hiện trong nghiên cứu trước cho thấy, EVA là một công cụ đa năng nhưng hạn chế trong các nghiên cứu này là do đa số đối tượng khảo sát là các doanh nghiệp trong lĩnh vực sản xuất. Câu hỏi đặt ra là liệu EVA có thực sự hữu hiệu khi vận dụng vào trong các ngành, lĩnh vực khác như nông nghiệp, giáo dục, $\mathrm{y}$ tế và du lịch,...v.v. Một số nghiên cứu đã mở rộng phạm vi nghiên cứu sang lĩnh vực du lịch, nông nghiệp và cho thấy, EVA cũng hữu ích trong các lĩnh vực này ví dụ, Kim (2006) đã nghiên cứu ảnh hưởng của EVA và các thước đo truyền thống đến lợi nhuận của các doanh nghiệp kinh doanh dịch vụ khách sạn. Kết quả nghiên cứu chỉ ra rằng, EVA tác động cùng chiều với lợi nhuận của doanh nghiệp; Geyser và Liebenberg (2003) đã khảo sát thực trạng vận dụng EVA trong các doanh nghiệp kinh doanh nông nghiệp và các hợp tác xã ở Nam Phi và đã cung cấp bằng chứng thực nghiệm chỉ ra rằng, tồn tại thước đo EVA trong việc đánh giá thành quả hoạt động đối với các doanh nghiệp này. Kết quả trên hàm ý rằng, vẫn còn những ngành nghề, lĩnh vực khác cần tiếp tục nghiên cứu vai trò và tầm quan trọng trong việc ứng dụng thước đo này.

\section{KẾT LUẬn}

Trên thế giới, rất nhiều nghiên cứu về EVA đã được thực hiện, sự đóng góp của EVA đối với giá trị tạo ra cho cổ đông và sự phù hợp của EVA cho nhiều ngành khác nhau (Evanomics, 2011), tuy nhiên bên cạnh đó cũng có một vài nghiên cứu có quan điểm trái chiều hoặc cung cấp bằng chứng khác biệt. Tổng kết này đã nỗ lực để xem lại các nghiên cứu về EVA. Thông qua việc trình bày sơ đồ phân loại các nghiên cứu bằng cách phân loại các nghiên cứu thành bảy nội dung liên quan đến EVA như (1) Nghiên cứu liên hệ giữa EVA và biến động giá trị thị truờng (MVA), (2) Nghiên cứu tuoong quan giũa EVA và lợi tức cổ phiểu, (3) Nghiên cứu mối quan hệ giữa quản lý dụa trên giá trị (VBM) và EVA, (4) Nghiên cứu kỹ thuật tính toán EVA, (5) Nghiên cúu xây dưng khái niệm EVA và nguyên tắc vận dụng, (6) Nghiên cưu thực nghiệm về ảnh hưởng của EVA lên hành vi quản lý của nhà quản lý, (7) Nghiên cúu các úng dụng phát triển của EVA. Từ phân tích, so sánh và tổng kết các nghiên cứu trước liên quan đến EVA cho thấy hướng đi nghiên cứu tiếp theo:

Một là, cần tiếp tục nghiên cứu các vấn đề kỹ thuật trong việc tính toán EVA như lựa chọn các điều chỉnh trên các báo cáo tài chính phù hợp để đơn giản hóa kỹ thuật tính toán và loại bỏ được ảnh hưởng chủ quan trong việc lựa chọn các chính sách và ước tính kế toán chưa hợp lý;

Hai là, cần điều tra việc vận dụng EVA trong từng ngành hoặc lĩnh vực cụ thể để từ đó có thể tổng kết, đánh giá và hoàn chỉnh khái niệm $\mathrm{EVA}$, đặc biệt là cần có các nghiên cứu về $\mathrm{EVA}$ ở các nước đang phát triển.

Cuối cùng, nên mở rộng phạm vi nghiên cứu EVA thông qua việc xem xét các dữ liệu liên quan trong một khoảng thời gian dài hơn để kiểm tra mức độ hữu hiệu của thước đo này qua đó nâng cao khả năng ứng dụng trong việc đánh giá thành quả hoạt động của các tổ chức.

\begin{tabular}{|l|l|l|}
\hline \multicolumn{3}{|c|}{ DANH MƯC CÁC CHŨ VIẾT TÁT } \\
\hline \multicolumn{1}{|c|}{ Chữ viết tắt } & \multicolumn{1}{c|}{ Tiếng Anh } & Tiếng Việt \\
\hline EVA & Economic Value Added & Giá trịinh tế gia tăng \\
\hline MVA & Market Value Added & Giá trị thị trường gia tăng \\
\hline ROE & Return on equity & Thu nhập trên vốn chủ sở hữu \\
\hline RI & Residual Income & Thu nhập thậng dư \\
\hline NPV & Net Present Value & Giá trị hiện tại thuần \\
\hline PV & Present Value & Giá trị hiện tại \\
\hline DCF & Discounted Cash Flow & Chiết khầu dòng tiền \\
\hline EPS & Earning Per Share & Thu nhập trên cố phiếu \\
\hline NOTPAT & Net Operating Profit After Taxes & Thu nhập sau thuế và lãi vay \\
\hline IRR & Internal Rate of Return & Tỷ lệ hoàn vốn nội bộ \\
\hline
\end{tabular}




\section{TÀI LIỆU THAM KHẢO}

1. Acheampong, Y. J. \& Wetzstein, M. E. (2001). A comparative analysis of value-added and Traditional measures of performance: an efficiency score approach. [Online] Available: http://ageconsearch.umn.edu/handle/16715.

2. Anand, M., Garg, A. \& Arora, A. (1999). Economic value added: business performance measure of shareholder value. Management Accountant, 34(5), 351-356.

3. Anastassis, C. \& Kyriazis, D. (2007). The Validity of the Economic Value Added Approach: an Empirical Application. European Financial Management, 13(1), 71-100.

4. Anderson, A. M., Bey, R. P. \& Weaver, S.C. (2004). Economic Value Added adjustments: much to do about nothing?. Paper presented at Financial Management Association Annual Conference.

5. Athanassakos, G. (2007) Value-based management, EVA and stock price performance in Canada. Management Decision, 45(9), 1397-1411.

6. Anil K. Sharma \& Satish Kumar (2010). Economic Value Added (EVA) - Literature Review and Relevant Issues. International Journal of Economics and Finance, Vol. 2, No. 2; May 2010.

7. Chen, S. \& Dodd, J. L. (1997). Economic Value Added (EVA): An Empirical Examination of a New Corporate Performance Measure. Journal of Managerial Issues, 9(3), 318-333.

8. Costigan \& Lovata, 2002. Empirical Analysis Adopters of Economic Value Added. Management Accounting Research, Vol. 13, No. 2, June 2002.

9. Erasmus, P.D. (2008), “The Relative and Incremental Information Content of the Value Based Financial Performance Measure Cash Value Added (CVA)", Management Dynamics, Vol.17, No. 1, pp.2-15.

10. Ferguson, R. \& Lestikow, D. (1998). Search for the Best Financial Performance Measure: Basics Are Better. Financial Analysts Journal, 54(1), 81-85.

11. Ferguson, R., Rentzler, J. \& Yu, S. (2005). Does Economic Value Added (EVA) improve stock performance or profitability? Journal of Applied Finance, 15(2).

12. Ferguson, R., Rentzler, J. \& Yu, S. (2006). Trading Strategy on EVA and MVA: are they reliable indicators of future stock performance? The Journal of Investing, 15(4), 88-94.

13. Kim, G.W. (2006). EVA and Traditional Accounting Measures: which Metric is a better predictor of market value of hospitality companies? Journal of Hospitality \& Tourism Research, 30(1), 34-49.

14. Liapis, K.J. 2010. 'The residual value models: A framework for business administration'. European Research Studies, Volume XIII, Issue (1), 2010.

15. Lehn, K. \& Makhija, A. K. (1996). EVA and MVA: as performance measures and signals for strategic change. Strategy \& Leadership, 24, 34-4.

16. Lefkowitz, S.D. (1999). The Correlation between EVA and MVA of companies. MBA Dissertation, California State University.

17. Maditinos, I. D., Sevic, Z. \& Theriou, N.G (2006). The Introduction of Economic Value Added (EVA) in the Corporate World. Paper Presented at The International Conference: Innovation, Entrepreneurship, and Competitiveness in Balkan and Black Sea Countries, Kavala, Greece, November2-4, 2006.

18. Moujib Bahri Josée St-Pierre Ouafa Sakka. (2011). Economic value added: a useful tool for SME performance management. International Journal of Productivity and Performance Management, Vol. 60 Issue: 6, pp.603. 

VÀ CÁC VẤN ĐỂ LIÊN QUAN

19. Nikhil Chandra Shil (2009), Performa Patel, R., \& Patel, M. (2012). Impact of Economic value added (EVA) on Share price: A study of Indian Private Sector banks. International Journal of Contemporary Business Studies, 3(1), 24-34.

20. Nikhil Chandra Shil (2009). Performance Measures: An Application of Economic Value Added. International Journal of business and management, Vol.4, No 3.

21. Nufazil Altaf (2016). Economic Value Added or Earning: What explains market value in Indian film, Future Business Jounal, December 2016 2(2):152-166.

22. Nicolás Cachanosky \& Peter Lewin (2016). An Empirical Application Of The Eva Framework To Business Cycles. Future business Journal.

23. O'Byrne, S.F. (1996). EVA and Market Value. Journal of Applied Corporate Finance, 9(1), 116-125.

24. Pal, K. \& Sura, S. J. (2007). Economic value added and traditional performance measures: a review of academic and Empirical literature. Amity Journal of Business Strategy, 1(2), 110-120.

25. Peterson, P. P. \& Peterson, D.R. (1996). Company Performance and Measures of Value Added. The Research Foundation of the Institute of Chartered Financial Analysts, Charlottesville, VA.

26. Phillips, D. (2007). The Value of Economic Reality: Applying Economic Value Added. [Online] Available:http://digitalcommons.liberty.edu/cgi/viewcontent.cgi?article=1008\&context=honors.

27. Salaga Jakub, Bartosova Viera \& Kicova Eva (2015). Economic Value Added as a Measurement Tool of Financial Performance. Procedia Economics and Finance, 484 - 489.

28. Shen, Y., Zou, L., \& Chen, D. (2015). Does EVA performance evaluation improve the value of cash holdings? Evidence from China. China Journal of Accounting Research, 8(3), 213-241.

29. Sharma, A.K. \& Kumar, S. 2010. 'Economic Value Added (EVA): Literature review and relevant issues'. International Journal of Economics and Finance, 2(2): 200-221.

30. Tian, I.V., Keung, E. Y. \& Chow, F. Y. (2003). Economic Value Added in the Hong Kong listed companies: A preliminary evidence. Paper Presented in the International Congress on Modelling and Simulation (MODSIM 2003), at Townsville, Australia, July, 14-17, 2003.

31. Zamia, J. K., Turetsky, H.F. \& Cochran, B. (2005). The MVA-EVA Relationship: Separation of Market Driven Versus Firm Driven Effects. Review of Accounting and Finance, 4(1), 32-49.

32. Viera Berzakova, Viera Bartosova, Eva Kicova (2015). Modification of EVA in Value Based Management. Procedia Economics and Finance 26 (2015), 317 - 324.

33. Worthington, A. \& West, T. (2004).Australian Evidence Concerning the Information Content of Economic Value-Added. Australian Journal of Management, 29(2), 201-224.

34. Xin'e, Z., Ting, W., \& Yuan, Z. (2012). Economic Value Added for Performance Evaluation: A Financial Engineering. Systems Engineering Procedia, 5, 379-387.

35. Yvonne, J. A. (2001). A Comparative Analysis of Value-Added and Traditional Measures of Performance: An efficiency score approach. .[Online] Available:http:// papers.ssrn.com/sol3/papers.cfm?abstract_id=259200. 


\section{Phụ lục: Các nghiên cứu trên thế giới}

\begin{tabular}{|c|c|c|c|c|c|}
\hline $\begin{array}{l}\mathbf{T} \\
\mathbf{T}\end{array}$ & Tác giả & Năm & Dòng NC & $\begin{array}{c}\text { Phương pháp } \\
\text { luận NC }\end{array}$ & Đóng góp của nghiên cứu \\
\hline 1 & Anil K. Sharma & 2010 & & Mô tả & $\begin{array}{l}\text { Phân loại, xác định những khoảng trống trong } \\
\text { các nghiên cứu hiện có và đề xuất hướng } \\
\text { nghiên cứu trong tương lai }\end{array}$ \\
\hline 2 & Liapis & 2010 & Content $^{\mathrm{d}}$ & Thực nghiệm & Cung cấp các điều chỉnh cần thiết để tính EVA \\
\hline 3 & $\begin{array}{l}\text { Nicolás Cachanosky } \\
\& \text { Peter Lewin }\end{array}$ & 2016 & Content $^{\mathrm{a}}$ & Thực nghiệm & $\begin{array}{l}\text { Cho biết EVA hay thu nhập là chỉ tiêu tốt nhất } \\
\text { để giải MVA }\end{array}$ \\
\hline 4 & $\begin{array}{l}\text { Moujib Bahri, Josée } \\
\text { St-Pierre, Ouafa } \\
\text { Sakka, }\end{array}$ & 2011 & Content $^{\mathrm{g}}$ & Thực nghiệm & $\begin{array}{l}\text { EVA có thể giúp các nhà quản lý phát hiện ra } \\
\text { vấn đề và xác định nguyên nhân cải tiến }\end{array}$ \\
\hline 5 & $\begin{array}{l}\text { Qin Chen and } \\
\text { XiaoMei Zhang }\end{array}$ & 2011 & Content $^{\mathrm{g}}$ & Mô tả & $\begin{array}{l}\text { Sử dụng tiến trình phân cấp để xây dựng ma } \\
\text { trận để giải quyết trọng số của Bảng điểm toàn } \\
\text { diện EVA và tìm ra các yếu tố ảnh hưởng nhiều } \\
\text { nhất đến giá trị doanh nghiệp. }\end{array}$ \\
\hline 6 & $\begin{array}{l}\text { H.M. van der Poll, } \\
\text { N.J. Booyse, A.J. } \\
\text { Pienaar, S. Büchner } \\
\text { \& J. Foot }\end{array}$ & 2011 & Content $^{\mathrm{d}}$ & Mô tả & $\begin{array}{l}\text { Cung cấp tổng quan về việc thực hiện Giá trị } \\
\text { Kinh tế gia tăng (EVA) ở Nam Phi }\end{array}$ \\
\hline 7 & $\begin{array}{l}\text { Zhao Xinÿe, Wang } \\
\text { Ting, ZhengYuan }\end{array}$ & 2012 & Content $^{\mathrm{g}}$ & Thực nghiệm & $\begin{array}{l}\text { Giới thiệu phương pháp đánh giá thành quả } \\
\text { hoạt động mới sử dụng việc tạo ra giá trị làm } \\
\text { chỉ số cốt lõi và kết nối EVA với chỉ số đánh } \\
\text { giá tài chính truyền thống để thiết lập phương } \\
\text { pháp đánh giá hiệu quả mới cho các ngân hàng } \\
\text { thương mại }\end{array}$ \\
\hline 8 & Christian Kraus & 2013 & Content ${ }^{\mathrm{i}}$ & Mô tả & $\begin{array}{l}\text { So sánh các thước đo thành quả khác nhau } \\
\text { (EVA / RAROC vs. MCEV Earnings) được sử } \\
\text { dụng để quản lý giá trị trong kinh doanh bảo } \\
\text { hiểm phi nhân thọ và phi nhân thọ }\end{array}$ \\
\hline 9 & $\begin{array}{l}\text { Abdul Ghafoor } \\
\text { Awan, Kalsoom } \\
\text { Siddique \& Ghulam } \\
\text { Sarwar }\end{array}$ & 2014 & Content $^{\mathrm{a}}$ & Thực nghiệm & $\begin{array}{l}\text { Tìm thấy ảnh hưởng cảu EVA đối với lợi tức } \\
\text { cố phiếu của các công ty. }\end{array}$ \\
\hline 10 & $\begin{array}{l}\text { Shrikant } \\
\text { Krupasindhu } \\
\text { PANIGRAHI, } \\
\text { Yuserrie } \\
\text { ZAINUDDIN, } \\
\text { Azzlina AZIZAN }\end{array}$ & 2014 & Content $^{\mathrm{a}}$ & Thực nghiệm & $\begin{array}{l}\text { Cung cấp ma trận chiến lược tài chính dựa trên } \\
\text { EVA và quản ly̆ tăng trương doanh thu của các } \\
\text { công ty xây dựng có niêm yết trên TTCK }\end{array}$ \\
\hline 11 & $\begin{array}{l}\text { Viera Berzakova, } \\
\text { Viera Bartosova, } \\
\text { Eva Kicova }\end{array}$ & 2015 & Content $\mathrm{t}^{\mathrm{e}}$ & Mô tả & $\begin{array}{l}\text { Mô tả chỉ số định hướng giá trị phố biến nhất- } \\
\text { giá trị gia tăng kinh tế (EVA) và So sánh các } \\
\text { giá trị của các chỉ số được sử dụng làm phương } \\
\text { pháp xác định giá trị doanh nghiệp }\end{array}$ \\
\hline 12 & Ahmed, H. & 2015 & Content $^{\mathrm{a}}$ & Thực nghiệm & $\begin{array}{l}\text { Xác định mối quan hệ giữa thu nhập của doanh } \\
\text { nghiệp, giá trị kinh tế gia tăng và giá trị của cổ } \\
\text { đông của các ngân hàng Hồi giáo được lựa } \\
\text { chọn ở Bangladesh }\end{array}$ \\
\hline 13 & Shad and Lai & 2015 & Content $^{\mathrm{g}}$ & Thực nghiệm & $\begin{array}{l}\text { Đề xuất một khái niệm để kiểm tra tác động của } \\
\text { ERM đối với giá trị của doanh nghiệp thông }\end{array}$ \\
\hline
\end{tabular}



VÀ CÁC VẤN ĐỀ LIÊN QUAN

\begin{tabular}{|c|c|c|c|c|c|}
\hline & & & & & $\begin{array}{l}\text { qua phương pháp đánh giá hiệu quả kinh tế } \\
\text { (EVA) }\end{array}$ \\
\hline 14 & $\begin{array}{l}\text { Nicolás Cachanosky } \\
\& \text { Peter Lewin }\end{array}$ & 2016 & Content $^{\mathrm{g}}$ & Thực nghiệm & $\begin{array}{l}\text { Xem xét chỉ tiêu giá trị gia tăng về kinh tế } \\
\text { (EVA®) đế ước tính tác động của biến động lãi } \\
\text { suất đối với các doanh. }\end{array}$ \\
\hline 15 & Nufazil Altaf & 2016 & Content $^{\mathrm{a}}$ & Thực nghiệm & $\begin{array}{l}\text { Kiểm tra tuyên bố của Stern Steward \& } \\
\text { Company rằng EVA là thước đo thu nhập tốt } \\
\text { hơn thước đo truyền thống dựa trên thu nhập }\end{array}$ \\
\hline & $\begin{array}{l}\text { Chitra Gunshekhar } \\
\text { Gounderv\& } \\
\text { Dr.M.Venkateshwarlu }\end{array}$ & 2017 & Content $^{\mathrm{a}}$ & Mô tả & $\begin{array}{l}\text { Đề xuất tập trung vào EVA để phân tích giá trị } \\
\text { cổ đông của các ngân hàng. }\end{array}$ \\
\hline
\end{tabular}

\section{Ghi chú:}

Content $^{\mathrm{a}}=$ Mối quan hệ EVA - MVA (EVA-MVA relationship); Content ${ }^{\mathrm{b}}=$ EVA và lợi tức cổ phiếu (EVA and stock returns); Content $^{\mathrm{c}}=$ Hành vi của nhà quản lý và hiệu quả hoạt động (Managerial behavior $\&$ performance management); Content ${ }^{\mathrm{d}}=$ khái niệm EVA và kỹ thuật tính toán (EVA concept, criticism, applications and strategy); Content ${ }^{\mathrm{e}}=$ Quản lý dựa trên giá trị $($ Value management $) ;$ Content $\mathrm{f}^{\mathrm{f}}=\mathrm{Cá}^{\mathrm{c}}$ phương pháp chiết khấu - quan hệ EVA và NPV (Discounting approaches); Content ${ }^{\mathrm{g}}=$ EVA và các úng dụng phát triến của EVA (Frameworks and models).

Ngày nhận bài: 27/12/2017

Ngày chấp nhận đăng: 11/03/2018 